\title{
Simulación de sistemas energéticos como metodología para el desarrollo competencial en la asignatura Ampliación de Energía
}

\author{
Rafael Miróa, Teresa Barrachina a, Belén Juste ${ }^{a}$, Ricardo Sanchís ${ }^{a}$, María Josefa \\ Palomo $^{a}$, Alberto Escriváa, Carlos Guardiolab, Vicente Bermúdez ${ }^{b}$, José Galindob, \\ José Javier López ${ }^{\text {, Jaime Martín }}{ }^{\text {b }}$ Ricardo Novellab \\ a Depto. de Ingeniería Química y Nuclear rmiro@iqn.upv.es, tbarrachina@iqn.upv.es, \\ bejusvi@iqn.upv.es, rsanchis@iqn.upv.es, mpalomo@iqn.upv.es, aescriva@iqn.upv.es \\ bDepto. De Máquinas y Motores Térmicos carguaga@mot.upv.es, bermudez@mot.upv.es, \\ galindo@mot.upv.es, jolosan3@mot.upv.es, jaimardi@mot.upv.es, rinoro@mot.upv.es
}

\begin{abstract}
The implementation of the New Masters for the convergence to the EHEA has led to the appearance of new advanced subjects of marked technical and professional character in the new master's degrees.

The main objective of this project has been the development and experimentation of free software, under Matlab environment, for the preparation of a portfolio for the development of the competences of the Master's degree in Industrial Engineering, as well as the transversal competences of the subject of Advanced Energy and Thermal Machines.

The development and use of free mathematical software for the resolution of professional problems of design and analysis of real industrial energy systems will facilitate the student's work by its easy availability, but needs to be oriented on the resources and time available, as well as on the use and capabilities of the developed programs.
\end{abstract}

Keywords: Competencies, Simulation, Problems, Demonstrations, software, Matlab $^{\circledR}$.

\footnotetext{
Resumen

La implantación de los Nuevos Másteres para la convergencia al EEES ha llevado aparejada la aparición de nuevas asignaturas avanzadas de marcado carácter técnico y profesional en los nuevos másteres habilitantes.

El objetivo principal del presente proyecto ha sido el desarrollo y experimentación de software libre, bajo entorno Matlab, para la elaboración de un portafolio para el desarrollo de las competencias propias del título de Máster en Ingeniería Industrial, así como de las competencias transversales de la asignatura de Ampliación de Energía y Motores Térmicos.
} 
El desarrollo y empleo de software matemático libre para la resolución de problemas profesionales de diseño y análisis de sistemas energéticos industriales reales facilitará la labor discente del alumno por su fácil disponibilidad, pero necesita ser orientado sobre los recursos y tiempo disponibles, así como sobre el uso y capacidades de los programas desarrollados.

Palabras clave: Competencias, Simulación, Problemas, Demostraciones, software, Matlab ${ }^{\circledR}$.

\section{Introducción}

El trabajo llevado a cabo formó parte de un PIME en el curso 2016-2017 y se ha realizado en la titulación Máster Universitario en Ingeniería Industrial de la ETSII, concretamente en la asignatura Ampliación de Energía y Máquinas Térmicas durante el curso académico 2016-2017.

El número de alumnos involucrados es de 298 repartidos en 6 grupos de teoria de aula. Cada grupo se divide a su vez en 3 grupos de prácticas con un total de 18 grupos.

La asignatura está dividida en dos bloques. La docencia de un bloque es responsabilidad del departamento de Ingeniería Química y Nuclear (DIQN), mientras que la del otro bloque es responsabilidad del Departamento de Máquinas y Motores Térmicos (DMMT). El número de profesores de la asignatura es 12, que es el número de profesores que han colaborado en esta experiencia.

Las acciones propuestas en este proyecto tienen por finalidad investigar, experimentar y evaluar las competencias propias y transversales en la asignatura Ampliación de Energía y Máquinas Térmicas mediante el uso de una herramienta de software abierto y libre desarrollada con el objeto de estimular su utilización por parte del alumnado y así potenciar su capacidad de autoaprendizaje.

El proyecto se articula a lo largo de cuatro ejes: i) cálculos analíticos; ii) cálculos unidimensionales; iii) cálculos acoplados de transporte de masa, momento y energía, iv) aplicaciones a casos reales en aplicaciones industriales. Estos cuatro ejes permiten abarcar un gran número de competencias tanto específicas como transversales del Máster en que se engloba la asignatura, así como en la actividad profesional de la Ingeniería Industrial.

La finalidad última del proyecto es proporcionar los conocimientos teórico-prácticos necesarios para que los alumnos desarrollen herramientas de cálculo para el estudio de sistemas energéticos de una forma avanzada, en especial los que utilizan cambio de fase, y en concreto determinar posibles problemas de seguridad e integridad, y sean capaces a posteriori de aplicar la metodología aprendida directamente al cálculo, diseño y análisis de equipos industriales, así como a la validación de resultados, e incluso al desarrollo de nuevas herramientas de cálculo, tanto para producción como para investigación.

(cc) EY-NC-ND 2018, Universitat Politècnica de València

Congreso IN-RED (2018) 
Además, se pretende introducir en las clases nuevos recursos que se ajusten al temario como apoyo en la práctica de aprendizaje de estos software abierto. Los vídeos explicativos de apoyo, screencast, laboratorios virtuales y prácticas informáticas desarrollados pueden acercar al alumno al problema rápidamente debido al impacto audiovisual que tienen, ya que no hay que olvidar que las generaciones actuales están creciendo en un ambiente en que la transmisión de información es mucho más interactiva e informatizada que antes. Por otra parte, para el docente elaborar sus propios vídeos didácticos y laboratorios virtuales si así lo desea es todo un reto ya que requiere, competencias tecnológicas, un amplio dominio de la asignatura y mucha creatividad.

\section{Objetivos}

El objetivo principal de la innovación es la implementación de software de código abierto relacionado con la simulación de los sistemas energéticos, tanto como herramienta de apoyo a la enseñanza como de usos profesionales, dotando a los alumnos de competencias útiles en su futura vida profesional.

Este objetivo general se logra a través de los siguientes objetivos parciales orientados a la minimización de los inconvenientes que presentan las plataformas de simulación de software libre y código abierto:

O1) Introducir en asignaturas de las titulaciones el uso de las técnicas más actuales de simulación en análisis y diseño de sistemas energéticos profesionales.

O2) Seleccionar las tablas de materiales y modelos físicos y matemáticos disponibles en la literatura científica y profesional que satisfagan los criterios de rigurosidad necesarios para que estas herramientas reproduzcan satisfactoriamente el comportamiento físico de los ejemplos a desarrollar.

O3) Reducir el tiempo de aprendizaje de las herramientas desarrollando Ejemplos Base de casos industriales reales que permitan la internalización de las etapas que consta toda simulación/análisis/diseño. Los Ejemplos Base tendrán un referente físico reconocible y permitirá al alumno aplicar y combinar conceptos específicos de aplicaciones industriales.

Es importante destacar que estos objetivos docentes tienen en cuenta el nivel de conocimiento y habilidades de partida de los alumnos para evitar un excesivo coste docente adicional para alcanzar un determinado nivel base para iniciar el desarrollo de los ejemplos.

\section{Descripción del desarrollo de la innovación}

Para alcanzar los objetivos del proyecto, se ha seguido el siguiente plan de trabajo:

\section{Tarea I: Selección y documentación de software}

Esta tarea ha constado de dos etapas: 
1. Análisis de los códigos disponibles

2. Desarrollo de modelos en Matlab ${ }^{\circledR}$ de problemas reales

Tras valorar el software disponible, se optó por el uso de Matlab ${ }^{\circledR}$ y Excel ${ }^{\circledR}$ por las siguientes razones:

- $\quad$ Están disponibles para los estudiantes con acceso libre desde los ordenadores de la Universidad y desde los ordenadores propios puesto que la Universidad paga por estas licencias.

- Los estudiantes han utilizado estos programas en cursos anteriores por lo que tienen el nivel requerido en este curso.

- $\quad$ Estos programas se utilizan posteriormente en la vida profesional.

Una vez seleccionado el software se ha resuelto diferentes problemas reales con diferentes datos y modelos con estos códigos. El desarrollo de la programación, es decir, la organización en funciones, subrutinas, variables de entrada y salida, se ha realizado de manera sencilla para que sea fácilmente entendible por los alumnos y por cualquiera que desee ejecutar estos programas.

\section{Tarea II: Adaptación o Creación de Materiales Docentes}

Se han desarrollado los contenidos docentes y competencias de la materia y titulación a la que se destina este proyecto. Se ha procedido a la Adaptación de Materiales cuando se disponía de un material docente.

Por otro lado, se ha llevado a cabo la Creación de Materiales con objetivo principal de desarrollar los ejercicios, casos, ejemplos de simulaciones, etc., que se han resuelto previamente con el software y que no existían previamente.

Este material se ha puesto a disposición de los alumnos en PoliformaT y se ha utilizado en clase como parte de las actividades presenciales.

\section{Tarea III: Elaboración de Guías de Software}

Las etapas generales de las que consta por lo general todo programa de cálculo son: i) Preprocesado; ii) Solver; iii) Post-procesado. Con esta tarea se pretende elaborar los materiales necesarios (manuales, tutoriales, presentaciones, animaciones, etc.) para el correcto uso y maximizar el rendimiento de las simulaciones. Estos materiales deben explicar cada una de las etapas con tal de establecer correctamente el flujo de tareas a realizar para las simulaciones.

Las unidades seguirán la siguiente estructura general:

- Objetivos de la unidad.

- Introducción.

- Segmentos de información (desarrollo de la información).

(cc) EY-NC-ND 2018, Universitat Politècnica de València 
- $\quad$ Errores más comunes.

- Recuerda que...

- Bibliografía relevante.

También se incluye en este apartado la elaboración de las unidades didácticas en formato presentación (Power Point) con especial énfasis en realizar una exposición clara y estructurada.

Adicionalmente, se han elaborado materiales de apoyo docente necesarios. Estos materiales son presentaciones, esquemas de resolución de los problemas, esquemas uso del formulario. Además se han elaborado objetos de aprendizaje en forma de videos screencast.

A continuación se muestran algunos ejemplos de los materiales desarrollados:

- $\quad$ En la imagen siguiente se muestra uno de los módulos desarrollados en Excel para la simulación de Turbinas (bloque DMMT):

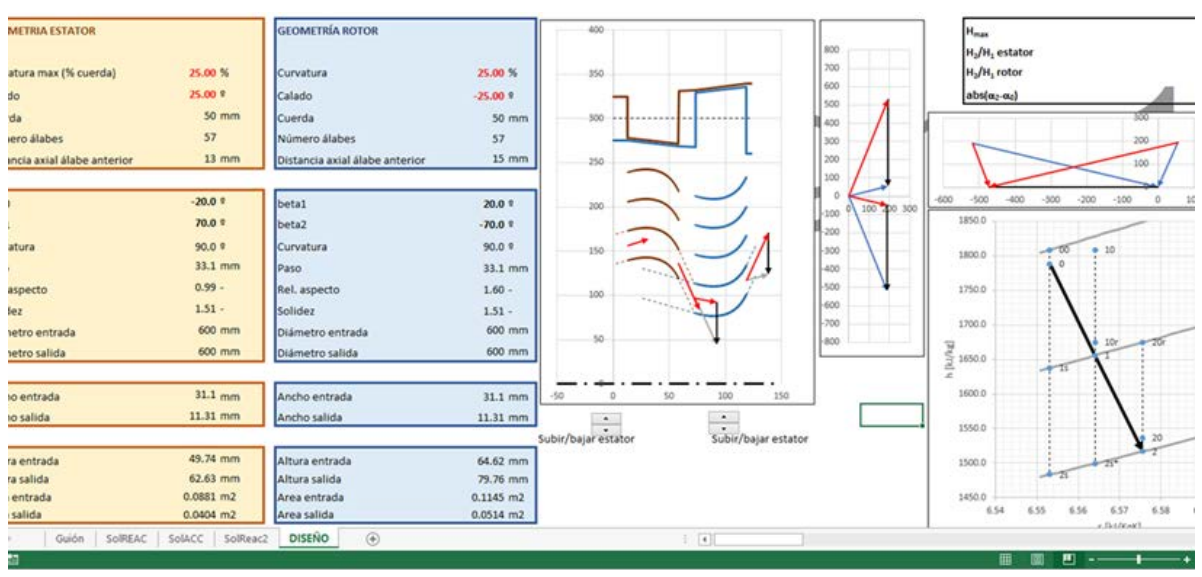

Fig. 1 Módulo en Excel para la simulación de Turbinas

- $\quad$ En la imagen siguiente se muestra uno de los diagramas de flujo para la resolución de los problemas complejos (bloque DIQN): 
Smulación de sistemas energéticos como metodología para el desarrollo competencial en la asignatura Ampliación de Energía.

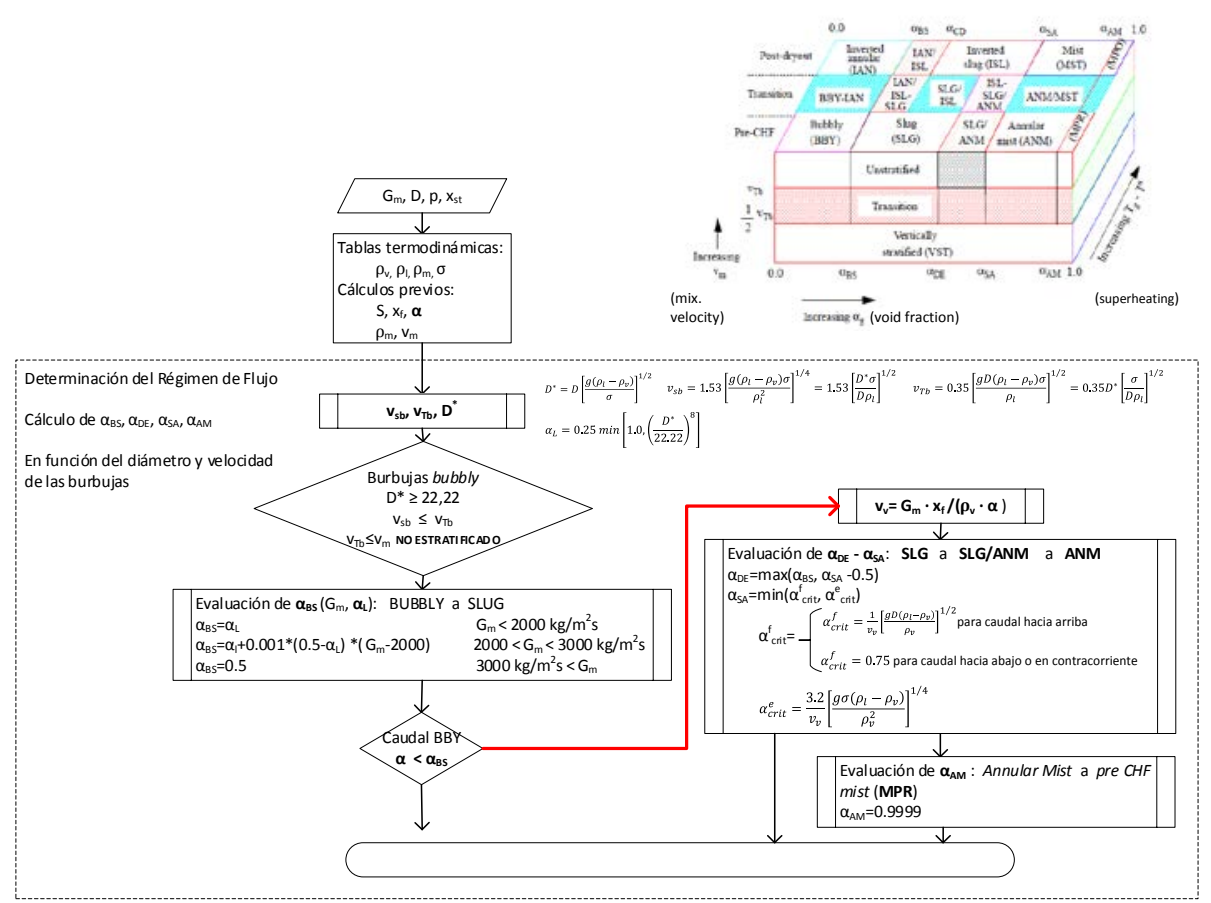

Fig. 2 Esquema del proceso de selección de régimen de caudal

- $\quad$ En la imagen siguiente se muestra uno de los programas desarrollados en Matlab (bloque DIQN):

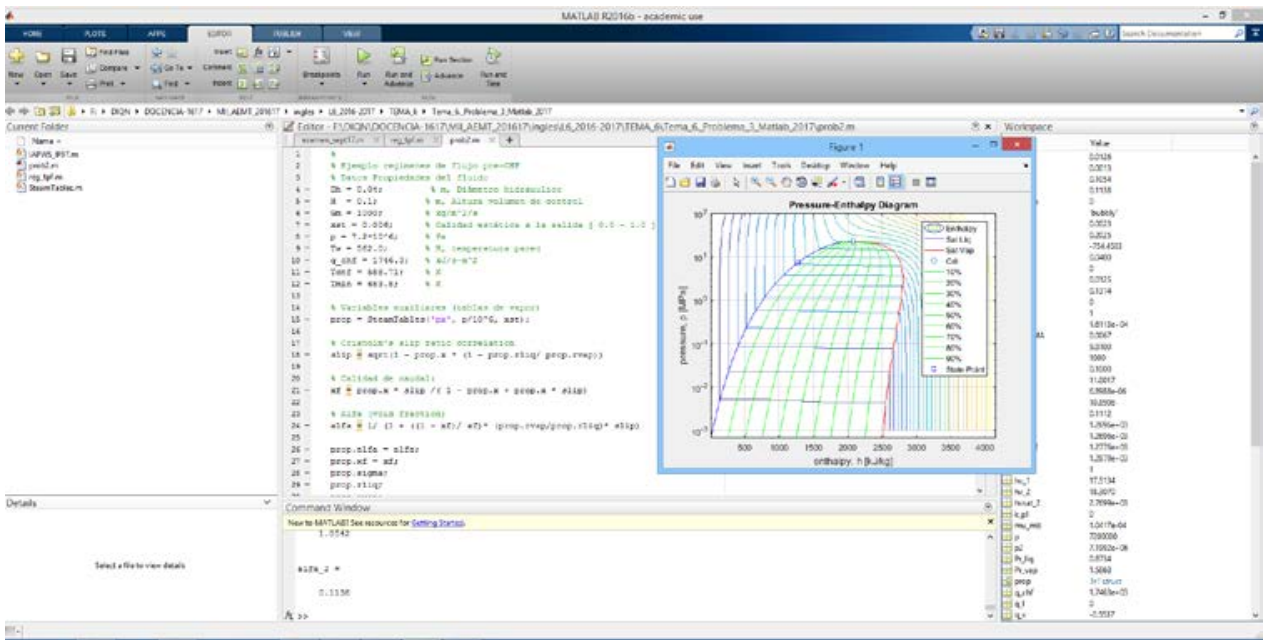

Fig. 3 Programa en Matlab para la resolución de los problemas

Además del diagrama presión entalpía, los alumnos también pueden obtener la curva de ebullición:

(c) EY-NC-ND 2018, Universitat Politècnica de València 


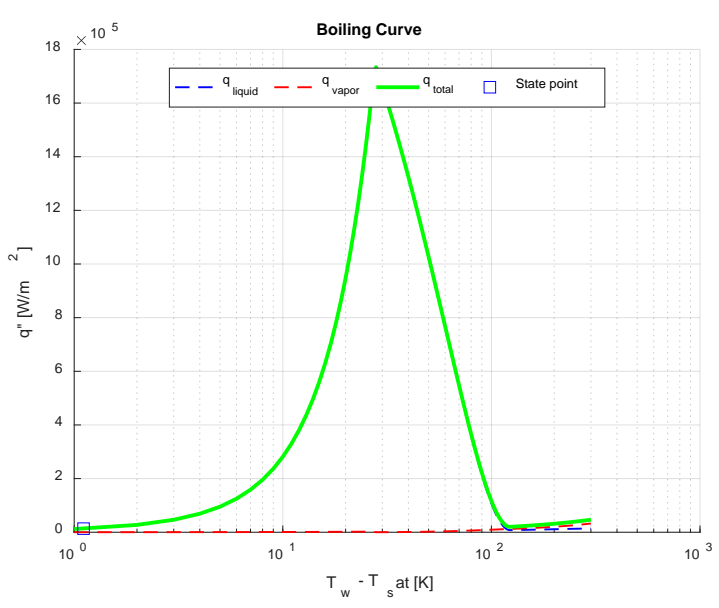

Fig. 4 Curva de ebullición obtenida con Matlab

\section{Resultados}

Se ha comprobado por testimonios de los alumnos que están deseosos de un cambio de la enseñanza con respecto a las prácticas tradicionales, y que al introducir elementos interactivos digitales en el curso, la respuesta de los alumnos ha sido muy satisfactoria.

Se han introducido mejoras de la comunicación educativa, lo que a su vez aumenta la motivación de sus estudiantes. Esto lo hemos podido constatar en las encuestas de opinión del alumnado.

Se ha constituido un factor de cambio en el modo de organizar el conocimiento de los alumnos. A través de los esquemas y diagramas de flujo, los alumnos tienen una nueva herramienta con la que interpretar el procedimiento de resolución de los problemas junto con el uso del software.

Se ha evaluado las competencias transversales a partir de las calificaciones obtenidas en los diferentes actos de evaluación. La evaluación de las CT se ha llevado a cabo de la siguiente manera:

\section{CT1 - Comprensión e integración}

Es la media aritmética de las calificaciones de las 2 preguntas de respuesta abierta de la evaluación del bloque del DIQN y de la calificación del test de la evaluación del bloque del DMMT.

\section{CT2 - Aplicación y pensamiento práctico}

Es la media aritmética de las calificaciones de las 2 memorias de las prácticas del bloque del DIQN y de las 2 prácticas del bloque del DMMT.

Las memorias de las prácticas se han evaluado utilizando una rúbrica que se adjunta al final de este documento. 
Smulación de sistemas energéticos como metodología para el desarrollo competencial en la asignatura Ampliación de Energía.

\section{CT3 - Análisis y resolución de problemas}

Es la media aritmética de las calificaciones de los problemas de la evaluación del bloque del DIQN y de los problemas de la evaluación del bloque del DMMT.

\section{CT13- Instrumental específica}

Es la calificación global de la asignatura.

Las calificaciones se han trasladado a la escala A, B, C, D dividiendo el rango de las calificaciones en cuatro partes iguales y asignando la escala A, B, C, D a cada parte en orden ascendente.

Por otro lado, la recogida de datos ha consistido en observación en el aula y testimonios de los alumnos. También se ha analizado las encuestas de opinión del alumnado y se ha comparado con años anteriores.

Los resultados de las calificaciones del curso 2015-2016 y del curso 2016-2017 se muestran en las figuras 5 y 6 :

\section{UNIVERSITAT}

POLITECCICA

DE VALÈNCIA

Estadísticas

Asignatura viernes 15 septiembre

33814 Ampliación de Energía y Máquinas térmicas (2015/2016)

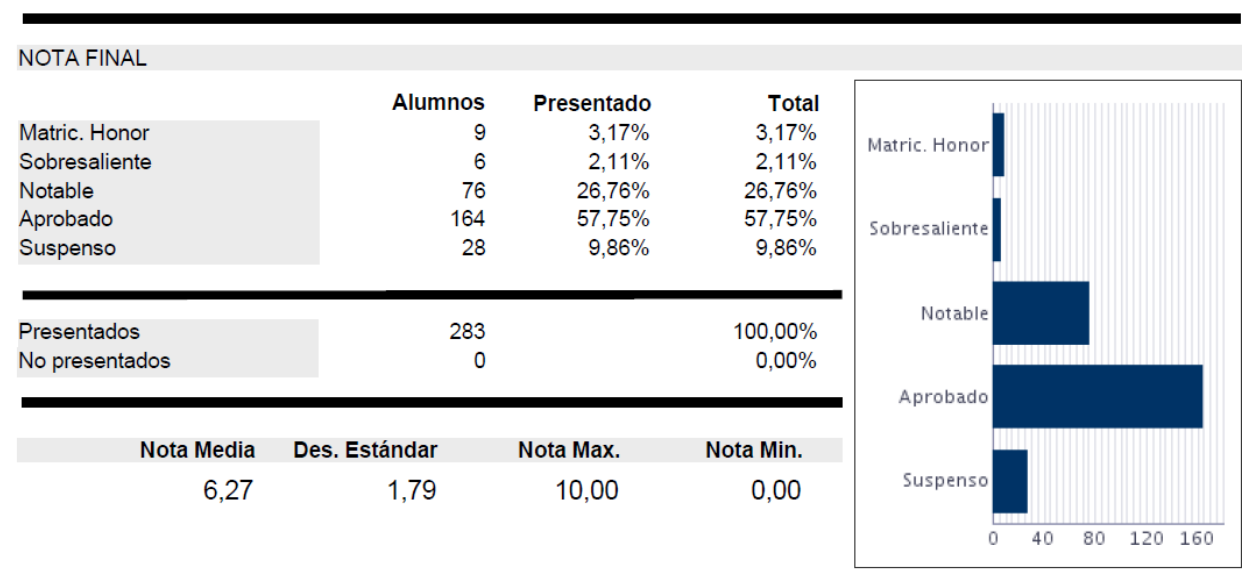

Fig. 5 Estadísticas de las notas del curso 2015-2016

(cc) EY-NC-ND 2018, Universitat Politècnica de València

Congreso IN-RED (2018) 


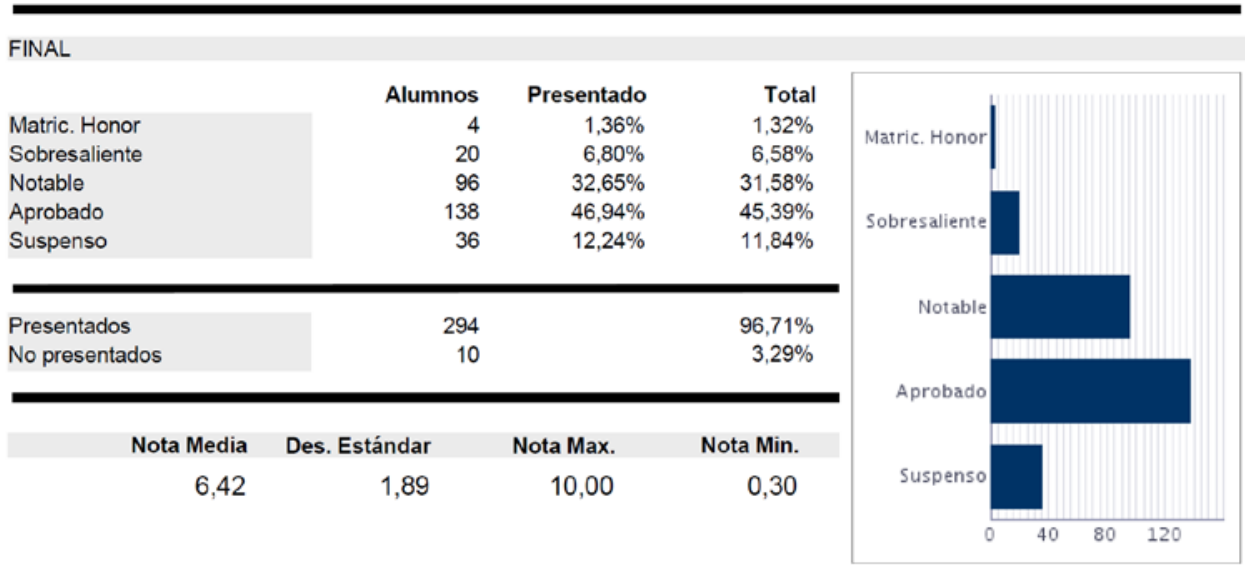

Fig. 6 Estadísticas de las notas del curso 2016-2017

Lo más destacable es el aumento de alumnos con Sobresaliente y Notable.

\section{Conclusiones}

El nivel de satisfacción derivado de la percepción positiva registrado en las encuestas junto con el rendimiento académico positivo alcanzado por estos estudiantes, avalan la utilización de las metodologías descritas.

En definitiva los resultados obtenidos han sido claramente positivos, aunque si tenemos en cuenta aspectos tales como los recursos y materiales utilizados, el curso mejorará en el futuro a medida que vayamos incorporando mejores materiales docentes (más laboratorios virtuales, screencasts, videos...) a la asignatura y el equipo docente adquiera experiencia también en el uso del software en el aula.

Consideramos además que la experiencia se puede transferir a cualquier otro contexto de la UPV, aunque nuestra recomendación es que se aplique en los últimos cursos de grado y los cursos de master, en los que el alumno ya cuenta con un determinado dominio de las herramientas informáticas y de cálculo.

\section{Referencias}

BABICH, A., MAVROMMATIS, K. (2009), “Teaching of Complex Technological Processes Using Simulations” en International Journal of Engineering Education, vol. 25, no 2, p. 209, 2009. 
Smulación de sistemas energéticos como metodología para el desarrollo competencial en la asignatura Ampliación de Energía.

CABERO, J. (2008), "Las TICs en la enseñanza de la química: aportaciones desde la Tecnología Educativa” en Química: vida y progreso. Asociación de químicos de Murcia, 2008.

IBRAHIM, D. (2011), "Engineering simulation with MATLAB: improving teaching and learning effectiveness” en Procedia Computer Science, vol.3, pp 853-858, 2011.

\section{Agradecimientos}

Los autores de este trabajo quieren expresar su gratitud al Instituto de Ciencias de la Educación y a la Escuela Técnica Superior de Ingenieros Industriales de la Universitat Politècnica de València, por su apoyo y financiación para la realización de este PIME concedido en el curso 2016-2017 (proyecto B25).

Este proyecto forma parte de los trabajos llevados a cabo por los miembros del EICE SEERI del Departamento de Ingniería Química y Nuclear.

(cc) EY-NC-ND 2018, Universitat Politècnica de València

Congreso IN-RED (2018) 